\title{
PHYSIOLOGICAL DIVERSITY AMONGST TRYPTOPHAN MUTANTS IN NEUROSPORA CRASSA
}

\author{
M. AHMAD* \\ Department of Botany, Dacca University, Eastern Pakistan \\ and \\ D. G. CATCHESIDE \\ Department of Microbiology, University of Birmingham
}

Received 23.xii.59

\section{INTRODUCTION}

THE discovery of complementation between alleles and especially the occurrence of patterns of relationship which can be reduced to linear complementation maps (Catcheside and Overton, I958 ; Pateman and Fincham, I958; Woodward, Partridge and Giles, I958) has made it advisable to examine a wide range of genes for the phenomenon. Two purposes prompt such a search, one that the frequency of occurrence is of interest and the other that instances more favourable for genetical and biochemical analysis may be found. Mutants affecting the biosynthesis of tryptophan are of especial interest in this connection. The genetic control of tryptophan synthetase is becoming extremely well understood and, indeed, after we had already gathered a large number of new tryptophan mutants, Lacy and Bonner ( 1958 ) reported the occurrence of complementation between some of the $t d$ mutants. They find that complementation appears to be restricted to those mutants, each of which produces a protein (CRM) which is sufficiently similar to tryptophan synthetase to cross-react with the specific antiserum to it.

It should be mentioned that the name of tryptophan synthetase has been changed from tryptophan desmolase, by which it was first known and after which the controlling gene was given the symbol $t d$. The gene has also been denoted tryp-3, as it was the third one discovered to affect tryptophan synthesis.

It has also become clear that indole glycerol phosphate is the compound from which tryptophan is formed, and that free indole is not itself an intermediate in synthesis (Yanofsky and Rachmeler, I958). It was suggested by Yanofsky and Rachmeler (I958) that synthesis. proceeds by the two coupled steps (I) indole glycerol phosphate+ tryptophan synthetase $\rightarrow$ indole tryptophan synthetase + triose phosphate. (2) indole tryptophan synthetase + L-serine $\rightarrow$ L-tryptophan. + tryptophan synthetase.

If these constituent processes are capable of independent loss by mutation, a variety of physiologically different mutants would be

* Work begun in University of Birmingham while holder of a Dominion Travelling Fellowship of the Nuffield Foundation. 
possible. Consequently, the discovery of $t d$ mutants able to utilise indole was not completely unexpected.

\section{MATERIAL AND METHODS}

A large number of mutants were obtained from the strain Emerson $a$ (5297) by a modified technique of filtration enrichment (Catcheside, 1954). The modifications were ( $\mathrm{I}$ ) the use of a macroconidial strain, a fair proportion of whose conidia are uninucleate, (2) agitation of the suspension by bubbling filtered air through the liquid medium, (3) use of Vogel's minimal medium (1956) containing a normal amount of sucrose but no sorbose and (4) maintenance of the cultures in a water bath at $25^{\circ} \mathrm{C}$. The filtered air was pumped through a Pasteur pipette inserted into the culture vessel (a boiling tube); to secure continued suspension of the conidia, and separately growing colonies, it was found necessary to have the orifice of the Pasteur pipette very close to the bottom of the tube. After the final filtration, suitable amounts of the suspension were plated in sorbose minimal medium, supplemented with $300 \mathrm{mg}$. tryptophan per litre. Colonies were isolated to individual tubes and tested on sorbose minimal medium to identify mutants. Tests of the ability of mutants to use anthranilic acid or indole were made on sorbose minimal medium supplemented with one of these compounds.

Heterocaryon tests were made by superimposing small drops of dense suspensions of conidia of the two strains to be tested with one another. When a large number of mutants had to be tested in all combinations, this was done on petri dishes containing sorbose minimal medium. Each dish was marked on the base with a grid of twenty-five squares, each half an inch square, and in five rows of five each. The individual dish and orientation of the square was identified by a code consisting of a letter and a number on adjacent sides. Small drops of conidial suspension were applied, a different strain to each horizontal row. When these drops had dried, they were overlaid with drops of other strains, a different one to each vertical row. In testing $n$ different mutants, all the $\frac{1}{2} n(n-1)$ combinations, as well as the $n$ control inoculations, would be set up within a relatively short time, using Pasteur pipettes with finely drawn nozzles to dispense the drops. In practice, not more than about fifty mutants can be managed conveniently in one such test. The plates were incubated at $25^{\circ} \mathrm{C}$ and examined at intervals for signs of growth. More critical tests were done singly on plates with control drops of the constituents.

\section{RESULTS}

A large number of mutants, well over ıoo, were isolated initially, but the number finally classified was only 77 (table I). The remainder included some which reverted to wild type and many which grew very poorly and were not worth the trouble of outcrossing to recover better stocks. Much less work would be involved in obtaining further mutants ab initio. It is evident that mutations deleterious to growth or conidial production are very frequent, but there is no indication that they are correlated with particular kinds of biochemical mutations.

The tryptophan mutants were first classified into the three nutritional groups (a) those (tryptophan mutants) which could grow only if tryptophan was supplied, (b) those (indole mutants) which could grow on indole as well as tryptophan and (c) those (anthranilic mutants) which could grow on anthranilic acid as well as on indole and tryptophan. Next, each nutritional group was further subdivided, if it could be, by heterocaryon tests. Initially, one mutant was selected at random in each group and tested for its ability to complement each of the others 
in the same nutritional group. All those with which it was negative would be judged as allelic with it. Those with which it was positive would then be further tested with one of their number in turn, and this process of division continued until all mutants in a nutritional group had been assigned.

These tests disclosed one group of tryptophan mutants, three groups of indole mutants and two groups of anthranilic mutants. Linkage tests and heterocaryon tests with standard tryptophan mutants showed that four of these six groups corresponded to previously known tryptophan mutants, but that two (one indole and one anthranilic) appeared to be new. Crosses were then made systematically to locate these presumed new genes.

The new indole group, represented by $\mathrm{A}_{45}$ and $\mathrm{A}_{7} 8$, was finally

TABLE I

Schedule of tryptophan mutants of Neurospora crassa. For full reference each number should be prefaced by a capital letter $A$.

$\operatorname{tryp}-\mathrm{I}$

group A A9, 48, 49, 51, 52, 56, 93, 94, I 05, I07, I I0, I 2 I, I 23

group B A20, 28, 38, 50,62, 63, I I 7

group $\mathrm{G}$ A $10,65,67,82$, I06

tryp-2

$\mathrm{A}_{4}, 55,60,64,80,8 \mathrm{I}, 95$, I 09 , I 12

$\operatorname{tryp-3}(=t d)$

group A Ai6, I 7, 2I-27, 29-34, 36, 41 , 43, 44, 46, 59, 68, 75,

group $\mathrm{B} \quad \mathrm{A}_{72}$

$76,79,88,89,97$, гоo, Iо I, Iо4, I I I, I 20, I 22

group $\mathrm{C} \mathrm{A}_{42}$, I 3

group D A $8,69,74,102$

group E A73

tryp-4

group $\mathrm{F} \mathrm{A}_{45}, 78$

located in chromosome II at a position which suggested it was closely linked to $t d(=t r y p-3)$. Each of these mutants were then tested for their ability to complement the $4^{2} t d$ mutants obtained in these experiments. It was found that each could complement 7 of the 42 , but none of the others. It was evident, therefore, that by heterocaryon tests $\mathrm{A}_{45}$ and $\mathrm{A}_{7} 8$ are allelic to all the other $t d$ mutants.

In view of the discovery (Yanofsky and Stadler, 1958) that some Escherichia coli mutants, which have a modified tryptophan synthetase, marked by inability to utilise indole glycerol phosphate, can nevertheless utilise indole in place of tryptophan, it was obviously possible that $\mathrm{A}_{45}$ and $\mathrm{A} 78$ were of this type and one of them was sent to Dr. Yanofsky. It has been reported (Rachmeler and Yanofsky, 1959) that $\mathrm{A}_{7} 8$ forms a tryptophan synthetase which can convert indole to tryptophan, but is inactive in the reactions involving indole glycerol phosphate. Antibody neutralisation tests, performed with the tryptophan synthetase of $\mathrm{A} 78$, have shown that it is inhibited to the same extent as is the enzyme of the wild type. 
The new anthranilic group, represented by Aio, A65, A67, A82 and Aio6, was located in chromosome III at a position very close to tryp-I (10575). Heterocaryon tests showed that they would complement some of our tryp-i mutants but not most of them.

Thus it was found that both of the new groups of mutants were allelic to two of the four tryptophan genes already known. Each of the new groups represents a new physiologically distinct type at its respective locus. These loci will now be considered in more detail.

\section{(i) Tryptophan-3}

This is better known as the tryptophan desmolase $(t d)$ or tryptophan synthetase locus. Our collection contains 44 mutants at this locus. When tested in all possible pairwise combinations (946) it was found that some pairs formed heterocaryons which would grow on minimal medium. Altogether ig pairs, involving io different mutants, showed complementation. The patterns of complementation between these

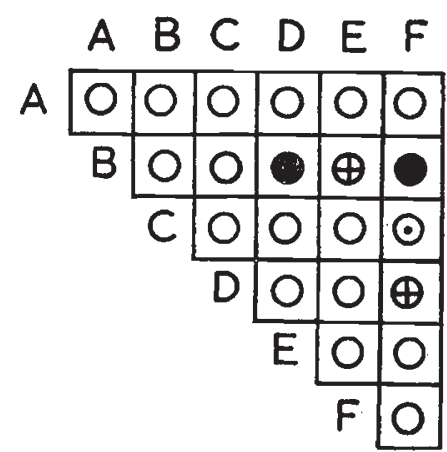

(a)

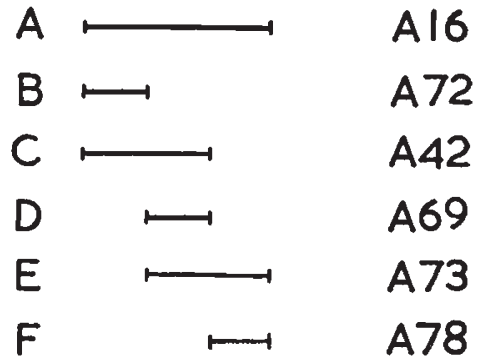

(b)

FIG. I.-Complementation between different groups of tryp-3 mutants. (a) Observed complementations : solid circle $=$ strong, inscribed cross $=$ moderate, inscribed dot = very weak, open circle $=$ no complementation. (b) Complementation map, with typical mutants.

ten mutants fall into five types. Including the large class of mutants, 34 in number, showing no complementation with any other allelic mutant, there are six patterns of reaction (fig. I $a$ ). This neglects the more subtle differences of vigour, which will be discussed later. The two-dimensional diagram can be represented as a one-dimensional complementation map (fig. $\mathrm{I} b$ ). The principle used in its construction is that each class of mutant is represented as inactivating a section of a linear structure so that noncomplementing ones overlap and complementing mutants do not overlap. The map drawn in figure $\mathrm{i} b$ is the only one possible that will represent the data of figure $\mathrm{i} a$ on these rules.

It is interesting to notice that class F, made up of the mutants which can grow on indole, comes at one end of the complementation map. It remains to be shown whether any of the mutants in classes $B, C$ and $D$, which are complemented by class $\mathrm{F}$ mutants, can carry out the 
reaction, indole glycerol phosphate $\rightarrow$ indole + triose phosphate. Nevertheless, it seems clear that there is some degree of regional specialisation within the tryp- $3^{+}$gene in respect of the two parts of the reaction converting indole glycerol phosphate + L-serine to L-tryptophan.

Amongst the nineteen heterocaryons that the ten complementing mutants can form, there is a considerable diversity in vigour. This is shown in two ways, one the speed of formation of a heterocaryon by superimposed drops of conidia and the other the growth rate or growth yield in a given time of an established heterocaryon on minimal

TABLE 2

Dry-weight yields of tryp-3 heterocaryons, grown in submerged liquid cultures, expressed as percentages of the yield of wild type; tr $=$ trace of growth, unweighed, probably about $\mathrm{O} \cdot \mathrm{I}$ per cent. of the weight of wild type.

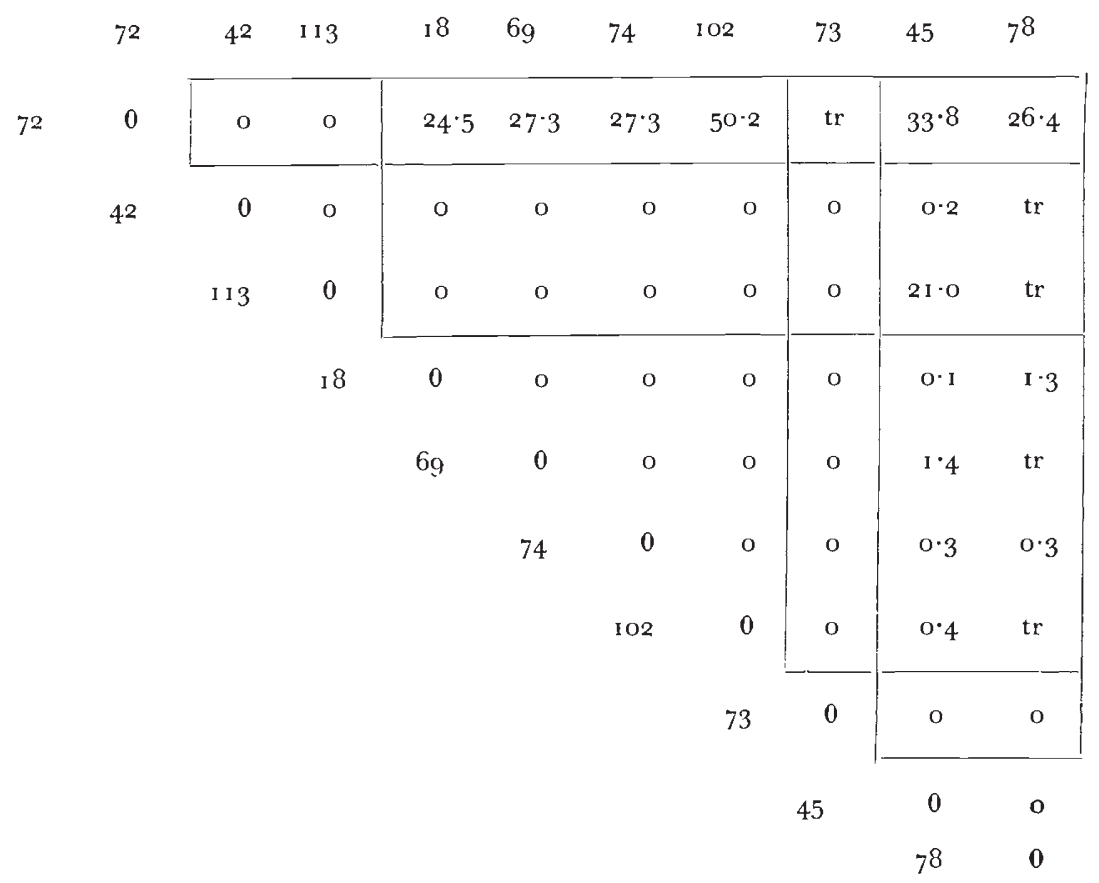

medium. The data shown in fig. I are intended to represent the vigour as assessed by speed of formation of a heterocaryon and its density of growth on solid medium. The growth yields have been determined in shaken conical flasks of minimal medium and expressed, in table 2, as percentages of the yield of wild type grown in the same way at the same time. The relative density of growth in submerged liquid culture does not always parallel growth on solid medium. Variation in vigour is shown not only between heterocaryons of different class relationship, but also between heterocaryons of the same class relationship. Thus of the eight heterocaryons possible between mutants of the $\mathrm{D}$ and $\mathrm{F}$ classes, $\mathrm{A}_{1} 8+\mathrm{A}_{7} 8$ and $\mathrm{A} 69+\mathrm{A}_{45}$ show some growth but 
the remaining six are very feeble. Amongst the heterocaryons possible between $\mathrm{A}_{72}$ and the four mutants of class $\mathrm{D}$, that involving $\mathrm{A}_{102}$ is about twice as vigorous as the other three. Thus the vigour of the heterocaryons, formed with group B and group F mutants, is a character which separates the four mutants of group D into subgroups.

\section{(ii) Tryptophan-1}

When it was discovered that the new group of anthranilic acid mutants, five in number, were allelic to tryp-I, we had 20 mutants already assigned to this locus. Heterocaryon tests showed that the anthranilic mutants (class C of fig. 2a) would complement seven of these (class B of fig. 2a). The heterocaryons were formed quite quickly; growth was often detectable at the end of one day and was well established in two days. The heterocaryons grew vigorously. They attained 83-9I per

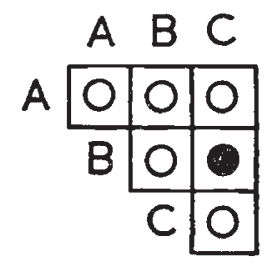

(a)

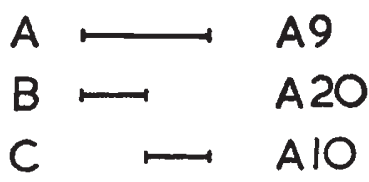

(b)

Fig. 2.-Complementation between different groups of tryp-I mutants. reactions. (b) Complementation map, with typical mutants.

(a) Observed

cent. of the dry-weight yield of wild type when grown in liquid minimal medium, on a shaker.

When tryptophan mutants are grown on slopes or in liquid medium in the presence of a limiting amount of tryptophan, the accumulation in the medium of substances that fluoresce in ultra-violet light is very marked. Mutants able to grow on tryptophan alone or both tryptophan and indole show a bright blue fluorescence. Mutants able to grow on anthranilic acid as well show a pale blue fluorescence.

If drops of culture filtrate are chromatographed, several substances may be distinguished, though most cannot yet be identified (table 3 ). The same compounds are accumulated by A2o (tryp-I of the B class) as by $\mathrm{A}_{5}($ tryp-4); these include anthranilic acid, the only compound identified positively. Mutant 10575 is probably also a member of class B. The mutant A io (tryp-i of the $\mathrm{C}$ class) and the mutants A9, $\mathrm{A}_{4} 8, \mathrm{~A}_{49}, \mathrm{~A}_{5} \mathrm{I}, \mathrm{A}_{52}$ and $\mathrm{A}_{5} 6$ (tryp-I of the A class) resemble $\mathrm{A}_{4}$ (tryp-2) in the detectable compounds they accumulate.

Thus the new type of tryptophan-I mutant (class G) differs from those previously known in three respects, (a) it can grow on anthranilic acid, (b) it can complement some, but not all, tryp-I mutants and (c) it does not accumulate anthranilic acid and the other compounds characteristic of tryp-I, but resembles tryp-2 in this respect. 


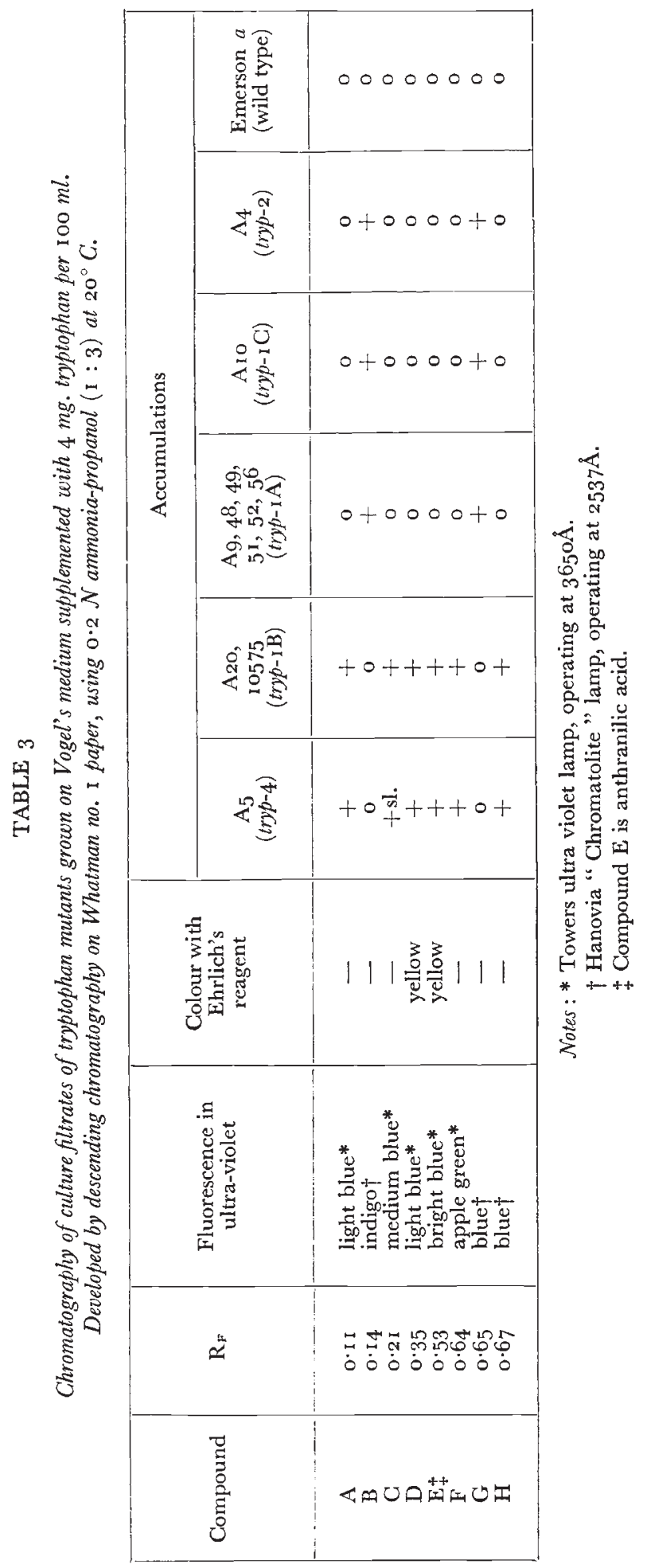




\section{DISCUSSION}

The present study has shown that two of the four genetic loci concerned specifically with the control of tryptophan synthesis are physiologically complex. We cannot say that the other two are not, because only a few mutants have been examined in each case, namely nine tryp-2 and four tryp-4.

We do not propose to discuss the general problem of the mechanisms of complementation, the outlines of which have been considered elsewhere (Catcheside and Overton, 1958). However, there is one aspect of the relation to function that is particularly appropriate to the present material. Roman (I958) and Yanofsky and Stadler (1958) have suggested that the complexities, of the kind shown by complementation. or by two related enzymic activities determined by the same gene, could be understood if two different products were controlled by overlapping segments of the chromosome (fig. 3). Mutations in the
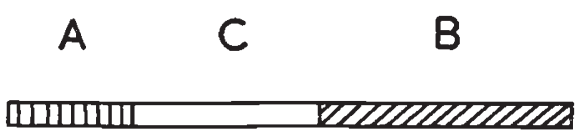

$\longleftrightarrow$ function $A \longrightarrow$

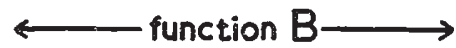

FIG. 3.-Diagram to show hypothesis of determination of two functions by overlapping segments of a chromosome.

common segment would be expected to eliminate both of the functions. simultaneously, while mutations in either of the unique segments would be expected to eliminate only the corresponding function.

In the case of the tryp-3 mutants, complete explanation along these lines is impossible because the gene behaves as though there were three distinct functions, any one or all of which can be eliminated by a single mutation. It is not possible to have a system of overlapping segments, involving three functions, such that each has a unique part of the linear genetic material and a segment common to all three. It seems more likely that the function is a unitary, if complex, one in which different genetic defects may cause more or less extensive physiological defects. Part of the function may be left, as in $\mathrm{A}_{7} 8$, or none, as in its allele Ar6.

Most of the $t d$ mutants accumulate indole glycerol rather than indole (Lacy and Bonner, 1958) despite that several of them produce the protein CRM which cross-reacts with the antibody to tryptophan synthetase. Only $t d^{2}$ and $t d^{71}$ accumulate indole; they belong to the same complementation class (Lacy, private communication). It may be that the altered enzymes, controlled by other mutants which produce CRM, while able to catalyse the reaction indole glycerol phosphate $\rightarrow$ indole + triose phosphate, leave the indole so tightly bound 
to the enzyme that the reaction is stopped. Perhaps $t d^{2}$ and $t d^{71}$ enzymes have a lowered affinity for indole and this results both in the accumulation of indole and in the prevention of the condensation of indole with $\mathrm{L}$-serine to form tryptophan. It has recently been shown (De Moss and Bonner, I959) that the partially defective enzyme produced by $t d^{71}$ requires pyridoxal phosphate to carry out the reaction indole glycerol phosphate + enzyme $\rightarrow$ indole-enzyme + triose phosphate, whereas normal tryptophan synthetase does not.

The genetic evidence suggests that tryp-I parallels tryp-3 $(=t d)$ in its physiological complexity. In the latter, indole is not a free intermediate in the biosynthesis of tryptophan. The substrate of the reaction is indole glycerol phosphate and indole is a bound intermediate in the reaction as a whole. Certain mutants, such as $\mathrm{A}_{7}$, are able to bind and use indole, but either cannot bind indole glycerol phosphate or else cleave it, because they have an altered tryptophan synthetase. The fact that $A_{1}$, and some other tryp-I mutants, can bind and use anthranilic acid, while other alleles (group A) neither use nor accumulate anthranilic acid, suggests that this substance is not a free intermediate in the normal reaction controlled by the gene tryp-I ${ }^{+}$. This reaction has been shown to involve a condensation between anthranilic acid and 5-phosphoribosyl-I-pyrophosphate (PRPP) to form a ribotide of anthranilic acid (Yanofsky, I956). It now appears that this is only a part of the reaction catalysed by the enzyme controlled by $\operatorname{tryp}-\mathrm{I}^{+}$. It seems necessary to look for some other compound, perhaps a phosphate of anthranilic acid or a similar substance, which would be bound by this enzyme and cleaved to yield an enzymeanthranilic acid complex which could then react with PRPP.

This evidence about the action of $\operatorname{try} p-\mathrm{I}^{+}$also indicates the relative

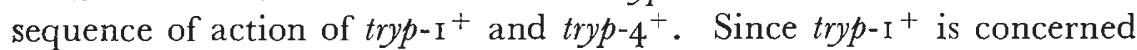
with the binding of anthranilic acid and tryp-4 mutants accumulate but cannot utilise anthranilic acid, the synthetic step controlled by tryp- $4^{+}$ must come after that controlled by tryp-I+ $\mathrm{I}^{+}$Tryp- $4^{+}$must be concerned with the binding of the ribotide of anthranilic acid, its rearrangement, the decarboxylation of the benzene ring and ring closure of the side chain to give indole glycerol phosphate. This appears to be a complex process and it would not be surprising to find that tryp-4 is also a complex locus.

\section{SUMMARY}

A set of 77 new tryptophan mutants, belonging to four genetic loci, have been classified physiologically. Complementation between alleles occurs amongst tryp-I and tryp-3 mutants. The complementation map of the tryp-3 (tryptophan desmolase) mutants is complex; two of the mutants are of a new type able to grow with indole as well as tryptophan. The complementation map of tryp-I is simple, but five of the mutants can grow with anthranilic acid. These five mutants do not accumulate anthranilic acid and other unidentified compounds, 
which are accumulated by complementing tryp-I alleles unable to utilise anthranilic acid. Non-complementing tryp-I alleles neither accumulate nor use anthranilic acid.

Acknowledgments.-We are indebted to Mrs Anne Hollis and Miss Angela Neale for technical assistance and to Miss Elisabeth Percy, who drew the diagrams.

\section{REFERENCES}

CATCHESIDE, D. G. I954. Isolation of nutritional mutants of Neurospora crassa by filtration enrichment. 7. Gen. Microbiol., II, 34.

Gatcheside, D. G. AND overton, Anne. i958. Complementation between alleles in heterocaryons. Cold Spr. Harb. Symp. Quant. Biol., 23, 137-140.

DE MOss, J. A., AND BONNER, D. M. I 959 . Studies on normal and genetically altered tryptophan synthetase from Neurospora crassa. Proc. Nat. Acad. Sci., Wash., 45, I405-1412.

LACY, ANNE M., AND BONNER, D. M. I958. Complementarity between alleles at the $t d$ locus in Neurospora crassa. Proc. X Int. Congr. Genetics, Montreal, 2, I 57.

pateman, J. A., And fincham, J. R. s. i958. Gene-enzyme relationships at the am locus in Neurospora crassa. Heredity, I2, 31 7-332.

RACHMELER, M., AND YANOFSKY, C. I959. Biochemical and genetic studies with a new $t d$ mutant type in Neurospora crassa. Bacteriol. Abstr.

Roman, H. 1958. Sur les recombinaisons nonréciproques chez Saccharomyces cerevisia et sur les problèmes posés par ces phénomènes. Ann. Génétique, I, I I-I 7 . vogel, H. J. I956. A convenient growth medium for Neurospora (Medium N). Microbial Genetics Bull., I3, 42-43.

WOODWARD, D. O., PARTRIDGe, C. W. H., AND GILES, N. H. I958. Complementation at the ad-4 locus in Neurospora crassa. Proc. Nat. Acad. Sci., Wash., 44, I 237 - 244.

YANOFSKY, C. 1956. The enzymatic conversion of anthranilic acid to indole. $\mathcal{J}$. Biol. Chem., 223, I 7 I.

YANOFSKY, C., AND RACHMELER, M. 1958. The exclusion of free indole as an intermediate in the biosynthesis of tryptophan in Neurospora crassa. Biochim. biophys. Acta, 28, 640-641.

YANOFSKY, C., AND STADLER, JOAN. I958. The enzymatic activity associated with the protein immunologically related to tryptophan synthetase. Proc. Nat. Acad. Sci., Wash., 44, 245-253. 